MANCHESTER

1824

部

Discussion Paper

Series

EDP-1813

\title{
Social Information and Consumer \\ Heterogeneity
}

Carlo Reggiani

Alejandro Saporiti

Lois Simanjuntak

October 2018

Economics

School of Social Sciences

The University of Manchester

Manchester M13 9PL 


\title{
Social Information and Consumer Heterogeneity*
}

\author{
Carlo Reggiani ${ }^{\dagger}$
}

Alejandro Saporiti

Lois Simanjuntak ${ }^{\S}$

\author{
September 2018
}

\begin{abstract}
We study the incentives of a social network to control two types of information circulating on its platform, namely display advertising by two quality-differentiated firms and "social information" (purchasing decisions shared among consumers). Consumers' choices are influenced by both of these communication channels, whereas the network gets revenue only through advertising. We characterize the equilibrium level of network diffusion of social information and the firms' expenditure on advertising. We show that depending on consumers' heterogeneous response to advertising, social information can compete with or complement display advertising. Moreover, in every equilibrium each consumer almost surely purchases the superior product with a strictly higher probability, and that receiving social information almost surely further increases such probability. Finally, social information almost surely raises social welfare, thereby establishing the existence of a welfare-maximizing equilibrium.
\end{abstract}

JEL Classification Codes: D82, D83, L12, L14, L15, L82.

Keywords: social information; display advertising; consumer heterogeneity; consumer (sequential) search; information transmission.

${ }^{*}$ We are grateful to James Banks, Marc Bourreau, Winand Emons, Kyriakos Neanidis, Antonio Nicolò, Giuseppe Pignataro, Chris Wallace and seminar participants at Manchester, the 2nd Doctoral Workshop on the Economics of Digitization (ParisTech), the IX IBEO Workshop on Media Economics (Alghero), the 45th EARIE Conference (Athens) and the XXXIII Jornadas de Economia Industrial (Barcelona) for useful comments and suggestions. The usual disclaimer applies.

†The University of Manchester; e-mail: carlo.reggiani@manchester.ac.uk.

†The University of Manchester; e-mail: alejandro.saporiti@manchester.ac.uk.

$\S$ The University of Manchester; e-mail: lois.simanjuntak@postgrad.manchester.ac.uk. 


\section{Introduction}

Online social networks are becoming more popular by the day: as of 2018 , there are 3.2 billion people using social media, that is forty two percent of the total population on the planet, up thirteen percent from 2017. Moreover, internet users are spending more time on social media: every day the average user spends two hours and fifteen minutes on them, accounting for one out of every three minutes spent online.

Giant networks like Facebook, YouTube, Instagram, LinkedIn, and Twitter are mostly "free" for their users ${ }^{1}$ As such, online advertising is a major source of income and a characterizing element of the business model of these platforms. Social media advertising is on the rise and networks are always looking for new advertising spaces in their websites and mobile apps.2 These sponsored messages aim to attract the attention of users to the products or services of their client firms.

Online advertising, however, is not the only tool that may raise users' awareness of products or services. Social network users, in fact, produce and share a huge amount of material ${ }^{3}$ In creating this mass of content, users may also endorse brands and share purchase choices with their social network's contacts. In other words, the "social information" circulating on a network may influence potential consumers in a way that is similar to advertising.

Importantly, social network users are likely to be heterogeneous in their reaction to each type of message. For example, marketing research suggests that "millennials" tend to be more influenced by social information and are less responsive to canonical advertising. 4 With the goal of amplifying the impact of online advertising on internet users, of attracting more advertisers, and of capturing a larger share of the marketing budgets, social networks employ sophisticated algorithms that control and orient the flow of "social information" circulating on their platforms.

\footnotetext{
${ }^{1}$ Facebook notoriously states on its initial page "It's free and it will always be".

${ }^{2}$ The forecast advertising revenue of social media for 2018 is about 51.3 billion USD, with an expected annual growth rate of 10.5 percent in the coming years, and it is predicted to almost double by 2023 . For further details about both the growth of social networks and the associated online advertising, see: https://blog.hootsuite.com/social-media-advertising-stats.

${ }^{3}$ During every minute of every day of 2014, according to Keen $(2015)$, the world's internet users uploaded 72 hours of YouTube video, shared $2.46 \mathrm{~m}$ pieces of Facebook content, published 277,000 tweets, and posted 216,000 new photos on Instagram, and these figures are likely to increase year after year.

${ }^{4}$ See: Newman, D. Research Shows Millennials Don't Respond To Ads. Forbes, April 28, 2015. Available at: https://www.forbes.com/sites/danielnewman/2015/04/28/ research-shows-millennials-dont-respond-to-ads/.
} 
In this context, the paper analyzes the incentives of a social network to control two kinds of potentially rival data circulating on its online platform: (a) display advertising, which consists of banners and other messages of uninformative content (such as text, images, flash, video, and audio) sent directly to the network users (consumers) by the advertisers (firms), and (b) social information, which takes the form of previous purchasing decisions shared among the consumers themselves on the network.

The model in this paper focuses on a social network connecting a continuum of consumers and two firms that sell quality-differentiated products. Consumers want to buy one of the products but the quality of each good is privately observed by sellers. Consumers can uncover quality before buying, by engaging in costly sequential sampling of the goods. Along with others, some examples of sampling include clicking on the banner or link that re-directs the consumer to the firm's web page, checking product specifications, opening comparison web-sites, reading reviews, or watching unboxing videos. In the model, diverse search costs reflect individuals' differences in the willingness to engage in such activities.

The network maximizes the firms' expenditure on display advertising. The main purpose of display advertising is to raise product awareness and increase the purchase intentions of consumers, but it is uninformative of the product quality. To be more precise, each firm uses its online advertisement to persuade the consumers to sample its product first. Besides sampling costs, consumers are also heterogeneous in their responsiveness to advertising, i.e., in the probability to be persuaded to sample a product.

Consumers do not buy the product all at the same time. This is captured by a mass of "early consumers" that buy the product first, and a mass of "late consumers" that shop afterwards. Besides sampling and shopping first, the early consumers observe on the network only display advertising, whereas each member of the late group, in addition to an advertisement, may independently observe online an early consumer's purchase. The probability of observing such information is strategically determined by the platform, and is referred to as the network diffusion of social information. The purchases of the early group provide a noisy signal about the quality of the products. The noise results because the late consumers do not know for sure if the early consumers have sampled one or both products, given the uncertainty about the individual costs of sampling (searching).

The paper provides the following results. First, we characterize the equilibrium level of network diffusion of social information and the firms' expenditure on advertising in our model. By doing that, we show that social information can compete with or complement display advertising. The nature of the relationship between these two ways of reaching consumers on the platform crucially depends on consumers' heterogeneous response to advertising. When consumers are equally responsive to advertising, the two information channels 
compete with each other: in equilibrium, the platform sets the minimum diffusion and shuts down social information to encourage firms' spending on advertising. When consumers react differently to advertising, display advertisements and social information can be complements. For that to happen, display advertising directly influences the more-responsive early consumers, while the transmission of their purchase decisions (social information) indirectly captures the late consumers, who are less responsive to advertising and are more likely to emulate their predecessors. In this case, the platform sets the maximum level of diffusion.

Moreover, we prove that in every equilibrium each consumer almost surely purchases the superior product with a strictly higher probability, and that receiving social information almost surely further increases the probability of buying such product. Finally, we show that social information almost surely raises social welfare, establishing as a by-product the existence of a welfare-maximizing equilibrium. These results confirm that, in our environment, social information is informative and valuable.

Our work is related to several streams of literature. First, recent work has focused on platforms, particularly in online markets. In the model we employ, the social network, like most platforms, plays the role of intermediary between unaware consumers and competing firms that advertise. Recent contributions have emphasized how such intermediaries can strategically distort the content provided to achieve revenue maximization: biased news are studied by Reuter and Zitzewitz (2006) and Ellman and Germand (2009) and biased search by Burguet et al. (2015) and de Cornière and Taylor (2014). The model in our paper shares this feature as the social network can strategically manipulate information diffusion.

Second, there is a rapidly growing literature on consumer search. Sequential search of different products was pioneered by Weitzman (1979) and recently used, inter alia, by Armstrong et al. (2009) and Armstrong and Zhou (2011) in studying "prominence" in search. In a sense, the display advertising in our setting helps firms achieve prominence. Mueller-Frank and Pai (2016) and Garcia and Shelegia] (2018) analyze sequential search and observational learning by agents, which are also important elements of the model in this paper.

Third, the literature on advertising has highlighted several roles of sponsored messages $5^{5}$ such as informative advertising and targeted advertising. For example, de Cornière \& de Nijs (2016) study the impact of targeting in a setting with price and match advertising on a platform. In our framework, online display advertising between competing firms has the sole objective of raising awareness: in particular, we adopt a form of all-pay auction to advertising expenditure developed by Friedman (1958) and more recently used in Bimpikis et al. (2016) and Dockner and Jørgensen] (2018).

Finally, our work also relates to the literature on information transmission in social

\footnotetext{
${ }^{5}$ See, e.g., Bagwel]. (2007), Renault] (2015), Choi et al. $[2017)$ for excellent reviews.
} 
networks. People, for example, are influenced by the opinions of friends in deciding which products to buy (Jackson!, 2014). They can also infer quality from the choices of others and/or acquire more information from peers (Kircher \& Postlewaite, 2008). Mueller-Frank and Pai (2015), the most closely related paper, shows that a social network has an incentive to block the transmission of social information in order to increase advertising revenue. The model in this paper builds upon theirs, with the advantage of comparability. Our main contribution is to emphasize the role of consumer heterogeneity in response to advertising and, by doing so, we show that it can be profitable for the network to allow full diffusion of social information. Therefore, their negative result of no transmission arises as a special case, when early and late consumers are identical in their responsiveness to advertising.

The rest of the paper is organized as follows. Section 2 describes the framework of firms, consumers, and the online social network. Our main results are presented in Section 3. We conclude the paper in Section 4, where we discuss the results and propose possible extensions. For expositional convenience, all proofs are in Appendix A.

\section{Setup}

Consider a market with two firms, indexed by $i=A, B$, who sell (at an exogenous price) a good of quality $q^{i} \in[0,1]$, where $\underline{q}=\min \left\{q^{A}, q^{B}\right\}$ (and respectively, $\bar{q}=\max \left\{q^{A}, q^{B}\right\}$ ) denotes the lowest (and respectively, highest) realized product quality. There is a continuum of consumers in the market divided into two groups $G=E, L$, referred to as the early and the late consumers, with typical elements denoted by $e \in E$ and $\ell \in L$, respectively. The mass of early consumers is normalized to 1 , and the (relative) mass of late consumers to $\lambda>0$.

To raise awareness of their products and maximize their sales (i.e., the number of units sold), the firms invest on display advertising (also known as banner advertising) in a social network (platform). The network maximizes the advertising expenditures of the firms by setting the diffusion $v \in[0,1]$ of information on the platform, which determines the probability that any late consumer independently observes the purchasing decision of an early consumer.

Both groups of consumers prefer the product with the highest quality. However, good quality is unobservable to the buyers. The early consumers shop first and carry out a costly sequential search; sampling a product perfectly reveals its quality. Consumers can sample either one or both products, before buying a single unit from one of the firms. The late consumers do the same afterwards. In addition to sampling, the late consumers might also receive social information circulating on the network, which consists of the purchases of 
the early consumers. The utility of each consumer is the quality of the purchased product minus the search cost.

Let $\alpha_{j}$ be the probability that consumer $j \in G$ samples first the product that she observes on the advertising banner. We interpret $\alpha_{j}$ as the responsiveness to advertising of consumer $j$ 's sampling decisions. That is, $\alpha_{j}$ reflects how influenceable and easy to persuade is $j \in G$ by the firms' advertising strategies. This persuasion parameter is heterogeneous across individuals and groups. The firms and the network know the mean values of $\alpha_{j}$ within each group $G=E, L$, but they do not know the exact value of $\alpha_{j}$ for each consumer $j \in G$. In the sequel, it is assumed that for each group $G=E, L$ and every consumer $j \in G, \alpha_{j}$ is independently drawn from a group-specific cumulative distribution function (c.d.f.) on $[0,1]$, with mean values $\hat{\alpha}_{G}>1 / 2$, and that it is privately observed by individual $j .^{6}$

The game of advertising and social information sketched above consists of the following sequence of events:

Period 0: The platform sets the diffusion $v \in[0,1]$ of the network. The quality $q^{i}$ of firm $i$ is independently drawn from a c.d.f. $F_{q}$ on $[0,1]$, with probability density $f_{q}$ positive everywhere. The firms observe the realized qualities $\left(q^{A}, q^{B}\right)$, and they simultaneously and independently choose the expenditures $m^{i} \in[0, M]$ on display advertising, where $M>0$ is a large positive integer. The probability that a consumer $j \in G$ independently sees a banner for product $A$, (and respectively, $B$ ) is given by the expenditure ratio $\rho=\frac{m^{A}}{m^{A}+m^{B}}$ (and respectively, $\left.1-\rho\right)$.

Period 1: The search cost $C_{E}$ of the early consumers is drawn from a c.d.f. $F_{c}$ on $[0,1]$, with density $f_{c}$ positive everywhere. Each early consumer $e \in E$ observes $\alpha_{e}$ and $C_{E}$, and with probability $\rho$ (and resp., $1-\rho$ ) she also observes a banner $\theta_{e}=A$ (and resp., $\theta_{e}=B$ ) of one of the firms. She subsequently samples at no $\operatorname{cost} \theta_{e}$ with probability $\alpha_{e}$, and the other product with probability $1-\alpha_{e}$. Sampling for the second time the remaining good is possible, but the cost is given by $C_{E}$. The quality $q_{s_{e t}}$ of the sampled products is immediately revealed, where $s_{e t} \in\{A, B\}$ denotes the sample choices of $e \in E$, with $t=1,1^{\prime}$ representing her two trials. The early consumer $e \in E$ buys the sampled product with the highest quality, with $a_{e} \in\left\{s_{e t}\right\}_{t=1,1^{\prime}}$ indicating her consumption choice.

Period 2: Each late consumer $\ell \in L$ engages into the same shopping routine than that of the early consumers, with $\alpha_{\ell}, C_{L}, \theta_{\ell}, s_{\ell t}, q_{s_{\ell t}}$, and $a_{\ell}$ redefined accordingly. The

\footnotetext{
${ }^{6}$ The fact that $\hat{\alpha}_{G}>1 / 2$ simply means that on average both groups sample first more frequently the product that appears on the ad. The main results of the paper do not rely on this assumption, and they extend easily to the case where either $\hat{\alpha}_{E}$ or $\hat{\alpha}_{L}$ are below half. Proofs are available upon request.
} 
difference between the groups is that before deciding which product to sample first, with probability $v$ consumer $\ell$ observes a purchasing decision $a_{e}$, which is randomly selected with equal probability from the set of the early consumers' purchased goods.

An equilibrium of the game of advertising and social information is a perfect Bayesian Nash equilibrium in pure strategies.

\section{Results}

Let $N \subseteq E \cup L$ represent the group of (early and late) consumers who only observe display advertising, and let $O \subseteq L$ be the set of late consumers who observe social information in addition to the advertisement. Note that a consumer $j \in N \cap G, G=E, L$, that samples first a product of quality $q^{i}$ will sample a second good if and only if the expected quality gain (according to $j$ 's prior belief) from the second trial is above the search $\operatorname{cost} C_{G}$ of the group to which $j$ belongs; that is,

$$
\int_{q^{i}}^{1}\left(q-q^{i}\right) d F_{q}(q) \geq C_{G} .
$$

Denote by $c_{N}\left(q^{i}\right)=\int_{q^{i}}^{1}\left(q-q^{i}\right) d F_{q}(q)$ the cut-off cost that makes individual $j \in N$ indifferent between searching once or twice, given that the quality of the product sampled first is $q^{i}$. The probability that $j \in N$ searches only once (buys the first product sampled $i$ ) is $\tau_{N}\left(q^{i}\right)=1-F_{c}\left(c_{N}\left(q^{i}\right)\right)$. For notational convenience, denote $\tau_{N}(\underline{q})=\underline{\tau}_{N}$.

Likewise, a consumer $j \in O \subseteq L$ that observes a purchase $a_{e}=i$ of quality $q^{i}$ reckons that either: (1) the early consumer $e$ could have sampled only $a_{e}$, which provides no valuable information, or (2) $e$ could have sampled both $A$ and $B$, in which case $a_{e}$ must be the highest quality good. Thus, individual $j$ 's posterior belief is that $a_{e}$ dominates (quality wise) the other product, and that is what $j$ samples first.7 Further, consumer $j \in O$ will sample a second good if and only if,

$$
\tau_{N}\left(q^{i}\right) \cdot \int_{q^{i}}^{1}\left(q-q^{i}\right) d F_{q}(q) \geq C_{L},
$$

\footnotetext{
${ }^{7}$ Specifically, conditional on $\bar{q} \in(0,1]$ being the highest realized quality, consumer $j$ 's expected quality of sampling for the first time the observed purchase $a_{e}=i$ is given by $\mathbb{E}\left(q^{i} \mid \bar{q}\right)=\tau_{N}\left(q^{i}\right) \cdot Q(\bar{q})+(1-$ $\left.\tau_{N}\left(q^{i}\right)\right) \cdot \bar{q}$, where $\mathbb{E}(\cdot \mid \cdot)$ stands for the conditional expectation operator, and for each $\bar{q} \in(0,1], Q(\bar{q})=$ $F_{q}(\bar{q}) \cdot\left(\int_{q \in[0, \bar{q})} q d F_{q}(q)\right)+\left[1-F_{q}(\bar{q})\right] \cdot \bar{q}$. Since $Q(\bar{q})<\bar{q}$, it follows that for all $\bar{q} \in(0,1], \mathbb{E}\left(q^{i} \mid \bar{q}\right)$ is always greater than the expected quality $Q(\bar{q})$ of sampling the product of the other firm $-i$.
} 
where the left-hand side of (2) is $j$ 's expected quality gain from her second trial (according to her posterior belief). As before, define by $c_{O}\left(q^{i}\right)$ and $\tau_{O}\left(q^{i}\right)$, respectively, the cut-off cost and the probability of stop searching after the first sample for the (socially) informed late consumers, with $\tau_{O}(\underline{q})=\underline{\tau}_{O}$. It is immediate from the inequalities (1) and (2) that $c_{O}\left(q^{i}\right)<c_{N}\left(q^{i}\right)$ for all $q^{i} \in[0,1]$, and consequently that $\tau_{O}\left(q^{i}\right)>\tau_{N}\left(q^{i}\right) !^{8}$

Define the following function $h(\cdot)$, which captures the consumer heterogeneous response to advertising within the late group, and it plays a key role in the subsequent analysis:

$$
h\left(\hat{\alpha}_{E}, \hat{\alpha}_{L}, \underline{\tau}_{O}\right)=\underbrace{\left(\hat{\alpha}_{L}-\frac{1}{2}\right)}_{\begin{array}{c}
\text { uninformed late } \\
\text { group's bias to ad }
\end{array}}-\underbrace{\underline{\tau}_{O} \cdot\left(\hat{\alpha}_{E}-\frac{1}{2}\right)}_{\begin{array}{c}
\text { informed late } \\
\text { group's bias to ad }
\end{array}} .
$$

Notice that the main determinants of this function are: (a) each group G's mean sample bias towards advertising $\hat{\alpha}_{G}-1 / 2$, and (b) the probability $\underline{\tau}_{O}$ that the informed group emulates the behavior (buys the same good) of the consumers observed on the network. The former results from the fact that, on average, consumer groups may react differently to display advertisements. This is relevant because the responsiveness of the informed late consumers depends on that of the early consumers. The latter arises since not all informed late consumers emulate the early ones, and $\underline{\tau}_{O}$ captures exactly such moderating effect ${ }^{9}$

Combining (a) and (b), the hyperplane $h(\cdot)$ shows that when the sample bias towards advertising is the same for both groups, the effective bias of the informed late consumers is smaller by a fraction determined by $\underline{\tau}_{O}$. The reason is that advertising can influence the late group not only directly through the banner, but also indirectly through the persuasion exerted on the informed consumers by the early group's purchases. In other words, $h(\cdot)$ can be seen as the difference in the effective responses of the late group between direct advertising via banners and indirect advertising via social information. When $h\left(\hat{\alpha}_{E}, \hat{\alpha}_{L}, \underline{\tau}_{O}\right)>0$ (and resp., $h\left(\hat{\alpha}_{E}, \hat{\alpha}_{L}, \underline{\tau}_{O}\right)<0$ ), the effective bias of the uninformed (and resp., informed) late consumers is relatively higher and it is more effective to reach them through direct (and resp., indirect) advertising. Instead, when $h\left(\hat{\alpha}_{E}, \hat{\alpha}_{L}, \underline{\tau}_{O}\right)=0$, both display ad, social information, and any mixture of them are equally effective to reach the platform's users.

As is pointed out above, the next proposition characterizes the equilibrium strategies of the platform and the firms, for the different regions determined by $h\left(\hat{\alpha}_{E}, \hat{\alpha}_{L}, \underline{\tau}_{O}\right)$.

\footnotetext{
${ }^{8}$ Notice that $c_{O}\left(q^{i}\right)$ and $c_{N}\left(q^{i}\right)$ may be equal if $\tau_{N}\left(q^{i}\right)=1$, which requires $q^{i}=1$. However, given that $f_{q}$ is well-defined and is positive everywhere on the support $[0,1], q^{i} \neq 1$ almost surely.

${ }^{9}$ Depending on whether the late consumers observe or not the purchases of the early group, their probabilities of buying the first product sampled are, respectively, $\underline{\tau}_{O}$ and $\underline{\tau}_{N}$, with $\underline{\tau}_{O}>\underline{\tau}_{N}$.
} 
Proposition 1 (Equilibrium Characterization) The equilibria of the advertising and social information game are such that the expenditures on display advertising are strictly positive and the same for both firms, i.e., $m^{A}=m^{B}(=m)>0$, and

(i) $v=0$ and $m=\frac{1}{2} \underline{\tau}_{N}\left[\left(\hat{\alpha}_{E}-\frac{1}{2}\right)+\lambda\left(\hat{\alpha}_{L}-\frac{1}{2}\right)\right]$, if $h\left(\hat{\alpha}_{E}, \hat{\alpha}_{L}, \underline{\tau}_{O}\right)>0$;

(ii) $v \in[0,1]$ and $m=\frac{1}{2} \underline{\tau}_{N}\left[\left(\hat{\alpha}_{E}-\frac{1}{2}\right)+\lambda\left(\hat{\alpha}_{L}-\frac{1}{2}\right)\right]$, if $h\left(\hat{\alpha}_{E}, \hat{\alpha}_{L}, \underline{\tau}_{O}\right)=0$; and

(iii) $v=1$ and $m=\frac{1}{2} \underline{\tau}_{N}\left(\hat{\alpha}_{E}-\frac{1}{2}\right)\left(1+\lambda \underline{\tau}_{O}\right)$, if $h\left(\hat{\alpha}_{E}, \hat{\alpha}_{L}, \underline{\tau}_{O}\right)<0$.

It is clear from Proposition 1 that, except in the knife-edge case (ii) where any level of network diffusion is optimal, the equilibrium of the game is unique.

Corollary 1 (Uniqueness) If $h\left(\hat{\alpha}_{E}, \hat{\alpha}_{L}, \underline{\tau}_{O}\right) \neq 0$, then the advertising and social information game has a unique equilibrium.

The different levels of diffusion and social information emerging from the three types of equilibria (i)-(iii) described in Proposition 1 are illustrated in Figure 1. These levels depend on the position of the locus $h\left(\hat{\alpha}_{E}, \hat{\alpha}_{L}, \underline{\tau}_{O}\right)=0$. To elaborate, suppose that the responsiveness to advertising $\hat{\alpha}_{L}$ of the average late consumer is relatively low, meaning that it lies below the upward-sloping dashed line $\left(h\left(\hat{\alpha}_{E}, \hat{\alpha}_{L}, \underline{\tau}_{O}\right)<0\right)$. In this case, social information is from the firms' viewpoint more effective to exert influence on the group. The network is aware of this, and it maximizes the firms' expenditures by linking the early consumers' shopping decisions to the sampling behavior of the late buyers, allowing social information to circulate freely on the platform (i.e., $v=1$ ). The unique equilibrium is consequently located on the grey-shaded region on Fig. 1 .

By contrast, when $\hat{\alpha}_{L}$ is above the dashed line $\left(h\left(\hat{\alpha}_{E}, \hat{\alpha}_{L}, \underline{\tau}_{O}\right)>0\right)$, the late consumers are relatively more responsive as a group to advertising. This means that the firms can persuade this group more effectively by reaching them directly with the banners, without relying on the flow of social information. That offers little incentives to the platform to allow a high level of connection between the purchases of both groups. In fact, the only type of equilibrium consists of the cross-shaded region on Fig. 1, where the information diffusion is $v=0$. The situation illustrated by the red-shaded area (on $\left.h\left(\hat{\alpha}_{E}, \hat{\alpha}_{L}, \underline{\tau}_{O}\right)=0\right)$, that is, the second type of equilibria, is a knife-edge case where any level of diffusion $v \in[0,1]$ will do as well.

The next corollary displays as a special case of Proposition 1 the equilibrium when $\hat{\alpha}_{E}=\hat{\alpha}_{L}$, showing that there is only one type of equilibrium, in which the network does not allow social information to circulate on its platform. Fig. 1 exhibits the result on the blue-shaded region, which arises from the intersection between the 45-degree line and the cross-shaded area. 


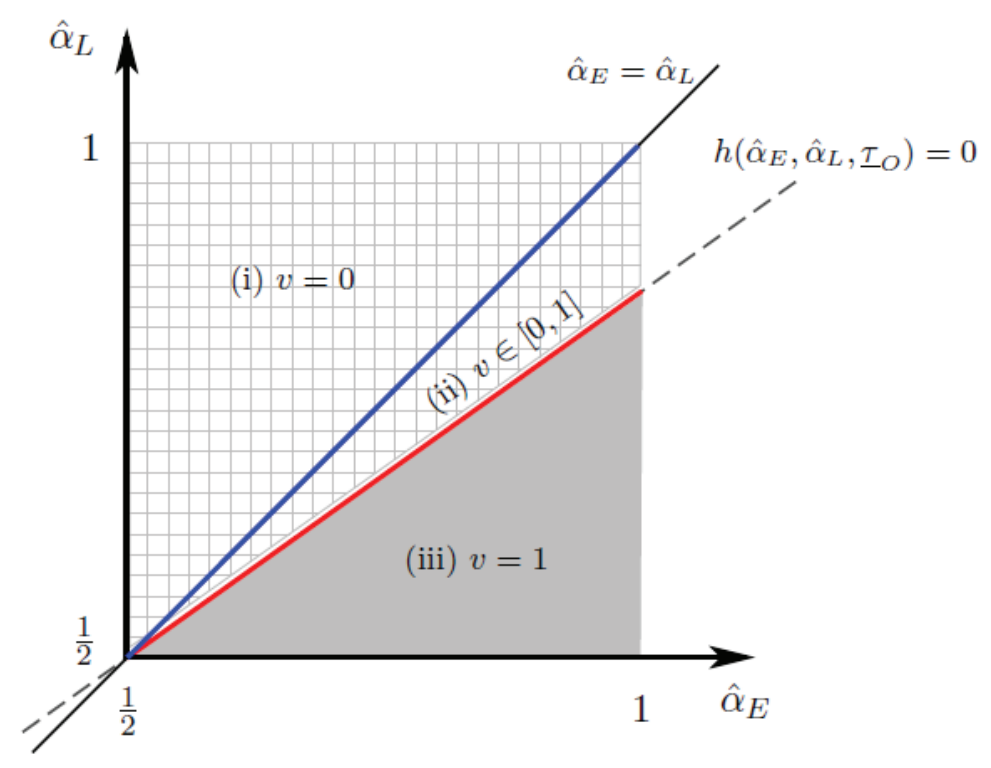

Figure 1: Social information and consumer heterogeneity

Corollary 2 (Mueller-Frank and Pai 2015) If $\hat{\alpha}_{E}=\hat{\alpha}_{L}=\hat{\alpha}$, then the equilibria of the advertising and social information game are such that the firms' expenditures are $m^{A}=$ $m^{B}=\frac{1}{2} \underline{\tau}_{N}\left(\hat{\alpha}-\frac{1}{2}\right)(1+\lambda)$, and the network diffusion is $v=0$.

Notice on Fig 1 the size of the equilibrium regions depends on the value of $\underline{\tau}_{O}$, which is the probability of an informed late consumer emulating an early one. When $\underline{\tau}_{O}$ is smaller, the posterior belief that the second sample is a better good is greater. The dashed line becomes flatter, and that enlarges the type (i) equilibrium region. This happens because it becomes more likely that the informed late consumers will sample twice and learn the true quality of the products, to which the platform reacts by closing the channel of social information in an attempt to increase the effective response of the late group to display ad. When $\underline{\tau}_{O}$ is larger, the posterior belief of the informed late group is, instead, smaller. The group does not expect much gain from a second trial, even after updating their beliefs with social information. As a result, it becomes more likely that the informed late consumers will buy the same product as the early consumer they observe on the platform. A greater $\underline{\tau}_{O}$ rises therefore the effective response of the late group to ad, by discouraging the informed subgroup to sample more than once. This shifts upwards the locus $h(\cdot)$ on Fig:1, and it expands the type (iii) equilibrium region, where social information circulates freely.

With regard to the firms' expenditures on advertising, Proposition 1 indicates that they are positive and identical for both firms, independent of the quality of their goods. That is, none of the firms (in particular, the high-quality firm) finds beneficial to signal the product 
quality to the consumers by spending more on display advertising. The reason why this happens is because consumers do not observe the firms' expenditures, but only a single banner with a certain probability. Hence, they cannot infer quality from $m^{i}$; and they cannot infer quality from $\theta^{i}$ either, since they do not know the frequency with which the banners are realized. That is, there is no way of truncating the distribution of quality after being exposed to a display ad. That implies that regardless of the quality of their products, both firms face exactly the same incentives (represented in Appendix A.1 by the first-order conditions (8) and (9)) to invest on advertising.

The closed-form expression for the equilibrium expenditures given in Proposition 1 offers the possibility of analyzing how display advertising varies with respect to the main parameters of the model, namely, $\mathbf{p}=\left(v, \hat{\alpha}_{E}, \hat{\alpha}_{L}, \lambda, \underline{\tau}_{O}, \underline{\tau}_{N}, q\right)$. The next proposition collects these results.

Proposition 2 (Comparative Statics) Let $m(\mathbf{p})$ denote the equilibrium expenditures of the firms in the advertising and social information game. Regardless of the network diffusion $v \in[0,1], \frac{\partial m(\mathbf{p})}{\partial p} \geq 0$ for each parameter $p=\hat{\alpha}_{E}, \hat{\alpha}_{L}, \lambda, \underline{\tau}_{O}, \underline{\tau}_{N}, \underline{q}$.

In accordance with the intuition, the above proposition confirms that at the equilibrium the firms' advertising expenditures are increasing in the groups' responsiveness to advertising, the fraction of late consumers, the probabilities of buying the first product sampled, and the lowest/minimum realized quality of the products. As to the comparison of $m$ for different values of the network diffusion $v$, the results are ambiguous. Equation (11) in Appendix A.1 shows that the sign of $\partial m / \partial v$ coincides with the sign of $-h\left(\hat{\alpha}_{E}, \hat{\alpha}_{L}, \underline{\tau}_{O}\right)$. Moreover, the advertising expenditures can be higher in either type of equilibrium depending mainly on the different values of $\hat{\alpha}_{E}, \hat{\alpha}_{L}$ and $\underline{\tau}_{O}$.

Turning to the consumer behavior, Appendix A.3 shows that in every equilibrium of Proposition 1, each consumer $j \in N$ who does not receive social information purchases the inferior product with probability $\frac{1}{2} \underline{\tau}_{N}$, which is the probability of observing the inferior good on the ad and buying the first product sampled. Similarly, each consumer $j \in O$ who does receive social information buys the inferior product with probability $\frac{1}{2} \underline{\tau}_{N} \underline{\tau}_{O}$, which is the probability that the early consumer buys the inferior good times the probability that the late consumer does not sample more than once. Thus, putting this together, it transpires that:

Proposition 3 (Consumer Behavior) In every equilibrium of Proposition 1, (1) each consumer almost surely purchases the superior product with a strictly higher probability, and (2) receiving social information almost surely further increases the probability of buying the superior product. 
In light of the results on consumer behavior, an interesting question to ask is whether social information increases society's well-being. To answer this question, define the ex-ante (expected) social welfare $W(\cdot)$ in the following way:

$$
\begin{aligned}
W(v)=\underbrace{\sum m^{i}}_{\text {network }}+\underbrace{\left(1+\lambda-\sum m^{i}\right)}_{\text {firms }}+\underbrace{1 \cdot \mathbb{E}\left(q_{a_{e}}-C_{E}\right)}_{\text {early consumers }}+ \\
\quad+\underbrace{\lambda \cdot\left[v \cdot \mathbb{E}\left(q_{a_{\ell}}-C_{L} \mid \ell \in O\right)+(1-v) \cdot \mathbb{E}\left(q_{a_{\ell}}-C_{L} \mid \ell \in N \cap L\right)\right]}_{\text {late consumers }},
\end{aligned}
$$

where $\mathbb{E}(\cdot)$ denotes the expectation operator.

Notice that the revenue of the network $\sum m^{i}$ is equal to the firms' expenditures on display advertising. In addition, the well-being of the early consumers $\mathbb{E}\left(q_{a_{e}}-C_{E}\right)$ is not affected by social information. Thus, rewriting (4) as

$$
\begin{aligned}
W(v)=1+\lambda+ & \mathbb{E}\left(q_{a_{e}}-C_{E}\right)+\lambda \cdot \mathbb{E}\left(q_{a_{\ell}}-C_{L} \mid \ell \in N \cap L\right)+ \\
& +v \cdot \lambda \cdot\left[\mathbb{E}\left(q_{a_{\ell}}-C_{L} \mid \ell \in O\right)-\mathbb{E}\left(q_{a_{\ell}}-C_{L} \mid \ell \in N \cap L\right)\right],
\end{aligned}
$$

it becomes apparent the social information diffusion $v$ only features in the last term of the right-hand side of equation (5). Hence, the efficiency of the three types of equilibria described in Proposition 1 depends on how the diffusion of the platform interacts with the difference between (a) the expected welfare (quality gain over the search cost) of the late consumers that observe the purchases of the early group, and (b) the expected welfare of those in the late group that decide which product to buy based solely on advertising information.

The next proposition points out that for the equilibrium of the advertising and social information game to maximize social welfare, the late consumers must be able to observe with certainty the purchases of the early group.

Proposition 4 (Social Welfare) In every equilibrium of Proposition 1, social welfare is almost surely strictly increasing in the network diffusion, i.e., $\frac{\partial W(v)}{\partial v}>0$. Therefore, $\arg \max _{v \in[0,1]} W(v)=1$.

An immediate implication of the above proposition is that only types (ii) and (iii) are welfare-maximizing equilibria. This happens because social information is valuable for consumers. There is a positive probability that an arbitrary early consumer has sampled both products, and therefore that the observed purchase, which is the first good sampled by the late consumers, has the best quality. Thus, any late consumer has a higher probability of purchasing the superior product if she receives social information. Moreover, since the late 
consumers are able to update their beliefs on quality with the information observed from the early buyers, their cut-off cost is lower and, consequently, they spend less on search.

\section{Concluding remarks}

This paper studies the incentives of an online social network to control two kinds of data circulating on its platform: display advertisement and social information. Online social networks accrue revenues through advertising and their users are potential buyers of the advertised products. Social information is produced by users posting on the network and it may be useful to other consumers. Since such sharing may include purchase choices, online social information is potentially a substitute to conventional advertising in raising consumers' awareness, and its flow can be controlled by the social network.

In this context, we build upon a platform model in which an online social network connects firms and consumers. The products of the two firms are exogenously differentiated in terms of their quality. Firms can invest in advertising but such expenditure cannot signal quality. In addition to having different search costs and time of purchase, in the model consumers are also heterogeneous in the level of responsiveness to advertising. We emphasize the role of such heterogeneity in determining the relationship between advertisement and the flow of social information, and how the latter can be distorted as a result.

The main results are as follows. First, if early and late consumers are, on average, fairly homogeneous in their responsiveness to advertising, then social information simply competes with sponsored advertising. Clearly, this damages profitability, so the social network has an incentive to limit the spread of social information. Heterogeneity, instead, can lead to a complementary relation between display advertisement and social information, so that the firms invest in advertising despite such information circulating on the platform. Complementarity happens when advertising reaches the more-responsive early group of consumers and their purchase decisions are then relayed, through social information, to the less receptive late group. Second, we have shown that social information almost surely increases the expected social welfare. Prospective consumers benefit from receiving information about previous consumers' choices through the network, as this increases the probability of buying the higher quality product. Finally, as a consequence of the two above findings, we establish the existence of welfare-maximizing equilibria in which a social network allows the circulation of social information on its online platform.

The conclusions, however, should not be overemphasized, as they were obtained in a rather stylized model of social networks. First of all, the model considers a unique platform. Whereas the assumption of monopoly is justifiable in situations with strong network effects 
or widespread multi-homing, the recent literature has emphasized the role of competition on more canonical offline media (Ellman \& Germand, 2009). In those environments, competing media platforms affect the quality of information provision to the audience. More recently, Prat and Valletti] (2018) model multiple social media platforms that have proprietary information about their users and sell targeted advertising to firms, and study the impact of platform mergers on the entry of new firms. Similar approaches on modeling competing online networks could be useful to study the spread of social information.

Second, we considered the platform to be an unbiased intermediary. In reality, however, commercial platforms may distort either the amount or the accuracy of information they display to the benefit of particular firms or political parties. Both the amount and accuracy of information can affect the users' decisions and welfare.

Third, the model is restricted to two groups of consumers and, consequently, only one comparison of responsiveness levels. This led to equilibria characterized by extreme values of information diffusion set by the network: all or nothing. Introducing more groups of consumers in the analysis may generate interior solutions, in which the platform lets circulate some optimal level of social information. A crucial aspect, however, is whether or not the social network can credibly commit to a single level of information diffusion to apply to all consumer groups.

Finally, more elements of the network structure could be incorporated in our model. According to Jackson (2014), the way people are connected in a network affects social learning. This relates to the notions of centrality, density, homophily, and the resulting clustering patterns. These elements are also likely to affect the platform's incentives to allow the flow of social information.

\section{References}

Armstrong, M. (2006). Competition in two-sided markets. The RAND Journal of Economics, $37(3), 668-691$.

Armstrong, M., \& Zhou, J. (2011). Paying for prominence. The Economic Journal, 121(556), F368-F395.

Armstrong, M., Vickers, J., \& Zhou, J. (2009). Prominence and consumer search. The RAND Journal of Economics, 40(2), 209-233.

Bagwell, K. (2007). The economic analysis of advertising. Handbook of industrial organization, 3, 1701-1844. 
Bimpikis, K., Ozdaglar, A., \& Yildiz, E. (2016). Competitive targeted advertising over networks. Operations Research, 64(3), 705-720.

Burguet, R., Caminal, R., \& Ellman, M. (2015). In Google we trust?. International Journal of Industrial Organization, 39, 44-55.

Choi, H., Mela, C. F., Balseiro, S., \& Leary, A. (2017). Online display advertising markets: A literature review and future directions. Columbia Business School Research Paper, 18-1.

de Cornière, A., \& Taylor, G. (2014). Integration and search engine bias. The RAND Journal of Economics, 45(3), 576-597.

de Cornière, A., \& de Nijs, R. (2016). Online advertising and privacy. The RAND Journal of Economics, 47(1), 48-72.

Dockner, E. J., \& Jørgensen, S. (2018). Strategic rivalry for market share: a contest theory approach to dynamic advertising competition. Dynamic Games and Applications, 1-22.

Ellman, M., \& Germano, F. (2009). What do the Papers Sell? A Model of Advertising and Media Bias. The Economic Journal, 119(537), 680-704.

Friedman, L. (1958). Game-Theory models in the allocation of advertising expenditures. Operations Research, 6(5), 699-709.

Garcia, D., \& Shelegia, S. (2018). Consumer search with observational learning. The RAND Journal of Economics, 49(1), 224-253.

Jackson, M. O. (2014). Networks in the Understanding of Economic Behaviors. Journal of Economic Perspectives, 28(4), 3-22.

Keen, A. (2015). The internet is not the answer. Atlantic Monthly Press. New York, NY, USA.

Kircher, P., \& Postlewaite, A. (2008). Strategic Firms and Endogenous Consumer Emulation. Quarterly Journal of Economics, 123(2), 621-661.

Mueller-Frank, M., \& Pai, M. (2015). Do Online Social Networks Increase Welfare? IESE Business School Working Paper, D/1118.

Mueller-Frank, M., \& Pai, M. M. (2016). Social Learning with Costly Search. American Economic Journal: Microeconomics, 8(1), 83-109. 
Prat, A. \& Valletti, T. M. (2018). Attention Oligopoly. Available at SSRN: https://ssrn.com/abstract $=3197930$.

Renault, R. (2015). Advertising in markets. In Handbook of Media Economics (Vol. 1, pp. 121-204). North-Holland.

Reuter, J., \& Zitzewitz, E. (2006). Do ads influence editors? Advertising and bias in the financial media. The Quarterly Journal of Economics, 121(1), 197-227.

Weitzman, M. L. (1979). Optimal search for the best alternative. Econometrica, 47(3), 641654 .

\section{A Appendix}

Since the probability density function $f_{q}$ of quality exists and is positive everywhere on the support $[0,1]$, the realized quality $q^{A}$ is almost surely different from the realized quality $q^{B}$. Thus, in the sequel assume without loss of generality that $q^{A}>q^{B}$.

\section{A.1 Proof of Proposition 1}

To start, note that the probability that an average consumer in group $N \cap G$ buys the lowest quality product is $P_{N}^{B}\left(m^{A}, m^{B}\right)=\left[1-\rho\left(m^{A}, m^{B}\right)\right] \hat{\alpha}_{G} \underline{\tau}_{N}+\rho\left(m^{A}, m^{B}\right)\left(1-\hat{\alpha}_{G}\right) \underline{\tau}_{N}$. Likewise, an average consumer in group $O \subseteq L$ buys good $B$ with probability $P_{O}^{B}\left(m^{A}, m^{B}\right)=$ $P_{E}^{B}\left(m^{A}, m^{B}\right) \underline{\tau}_{O}$. Thus, given the strategy of the platform $v \in[0,1]$, the conditional payoffs of firms $A$ and $B$ are, resp.,

$$
\Pi^{A}\left(m^{A}, m^{B}, v\right)=1+\lambda-\left\{\left[1+\lambda v \underline{\tau}_{O}\right] P_{E}^{B}\left(m^{A}, m^{B}\right)+\lambda(1-v) P_{N \cap L}^{B}\left(m^{A}, m^{B}\right)\right\}-m^{A}
$$

and

$$
\Pi^{B}\left(m^{A}, m^{B}, v\right)=\left[1+\lambda v \underline{\tau}_{O}\right] P_{E}^{B}\left(m^{A}, m^{B}\right)+\lambda(1-v) P_{N \cap L}^{B}\left(m^{A}, m^{B}\right)-m^{B} .
$$

Differentiating (6) and (7) with respect to firm $i$ 's own strategy $m^{i}$ and equalizing to zero, it follows that

$$
\underline{\tau}_{N} \frac{m^{B}}{\left(m^{A}+m^{B}\right)^{2}}\left\{\left[1+\lambda v \underline{\tau}_{O}\right]\left(2 \hat{\alpha}_{E}-1\right)+\lambda(1-v)\left(2 \hat{\alpha}_{L}-1\right)\right\}=1
$$

and

$$
\underline{\tau}_{N} \frac{m^{A}}{\left(m^{A}+m^{B}\right)^{2}}\left\{\left[1+\lambda v \underline{\tau}_{O}\right]\left(2 \hat{\alpha}_{E}-1\right)+\lambda(1-v)\left(2 \hat{\alpha}_{L}-1\right)\right\}=1 .
$$


It is easy to note that the system of first-order conditions defined by (8) and (9) has an interior solution if and only if $m^{A}=m^{B}=m>0$ The expression of the optimal expenditures $m(\cdot)$ of the two firms as a function of the diffusion $v$ is obtained by replacing $m^{A}=m^{B}=m$ back into the equations (8) and (9). To be more precise, defining $f\left(\hat{\alpha}_{E}, \hat{\alpha}_{L}, \lambda\right)=\left(\hat{\alpha}_{L}-1 / 2\right)+1 / \lambda\left(\hat{\alpha}_{E}-1 / 2\right)$, it follows that

$$
m(v)=\frac{\lambda}{2} \underline{\tau}_{N}\{f-v h\}
$$

where recall that $h\left(\hat{\alpha}_{E}, \hat{\alpha}_{L}, \underline{\tau}_{O}\right)=\left(\hat{\alpha}_{L}-1 / 2\right)-\underline{\tau}_{O}\left(\hat{\alpha}_{E}-1 / 2\right)$.

The conditional payoff of the network is $m^{A}(v)+m^{B}(v)=2 m(v)$. Substituting equation (10) and differentiating with respect to $v$,

$$
\frac{\partial(2 m)}{\partial v}=-\lambda \underline{\tau}_{N} h\left(\hat{\alpha}_{E}, \hat{\alpha}_{L}, \underline{\tau}_{O}\right)
$$

Thus, since $\lambda \underline{\tau}_{N}>0$, the sign of $\frac{\partial m}{\partial v}$ coincides with the sign of $-h\left(\hat{\alpha}_{E}, \hat{\alpha}_{L}, \underline{\tau}_{O}\right)$. To elaborate, if $h\left(\hat{\alpha}_{E}, \hat{\alpha}_{L}, \underline{\tau}_{O}\right)>0$, then $\frac{\partial m}{\partial v}<0$, and the best response for the platform is $v=0$. That means that $m=\frac{\lambda}{2} \underline{\tau}_{N}(f-v h)=\frac{\lambda}{2} \underline{\tau}_{N} f>0$ as required, where the last inequality follows from the restrictions on the mean values of $\alpha_{G}$, namely, $\hat{\alpha}_{G}>1 / 2$. Similarly, if $h\left(\hat{\alpha}_{E}, \hat{\alpha}_{L}, \underline{\tau}_{O}\right)=0$, then $\frac{\partial m}{\partial v}=0$, and any value of $v \in[0,1]$ is a best response to maximize the firms' expenditures on advertising. As before, $m=\frac{\lambda}{2} \underline{\tau}_{N} f>0$. Finally, if $h\left(\hat{\alpha}_{E}, \hat{\alpha}_{L}, \underline{\tau}_{O}\right)<0$, then $\frac{\partial m}{\partial v}>0$, which implies that the platform chooses $v=1$, and therefore $m=\frac{\lambda}{2} \underline{\tau}_{N}(f-h)>0$ as demanded. This completes the proof.

\section{A.2 Proof of Proposition 2}

The first-order partial derivatives of the equilibrium expenditures $m(\cdot)$ with respect to the parameters $\hat{\alpha}_{G}, \lambda, \underline{\tau}_{O}$, and $\underline{\tau}_{N}$ are as follows:

- $\frac{\partial m}{\partial \hat{\alpha}_{E}}= \begin{cases}\frac{1}{2} \underline{\tau}_{N}>0 & \text { if } h\left(\hat{\alpha}_{E}, \hat{\alpha}_{L}, \underline{\tau}_{O}\right) \geq 0 \\ \frac{1}{2} \underline{\tau}_{N}\left(1+\lambda \underline{\tau}_{O}\right)>0 & \text { if } h\left(\hat{\alpha}_{E}, \hat{\alpha}_{L}, \underline{\tau}_{O}\right)<0\end{cases}$

- $\frac{\partial m}{\partial \hat{\alpha}_{L}}= \begin{cases}\frac{1}{2} \underline{\tau}_{N} \lambda>0 & \text { if } h\left(\hat{\alpha}_{E}, \hat{\alpha}_{L}, \underline{\tau}_{O}\right) \geq 0 \\ 0 & \text { if } h\left(\hat{\alpha}_{E}, \hat{\alpha}_{L}, \underline{\tau}_{O}\right)<0\end{cases}$

- $\frac{\partial m}{\partial \lambda}= \begin{cases}\frac{1}{2} \underline{\tau}_{N}\left(\hat{\alpha}_{L}-\frac{1}{2}\right)>0 & \text { if } h\left(\hat{\alpha}_{E}, \hat{\alpha}_{L}, \underline{\tau}_{O}\right) \geq 0 \\ \frac{1}{2} \underline{\tau}_{N} \underline{\tau}_{O}\left(\hat{\alpha}_{E}-\frac{1}{2}\right)>0 & \text { if } h\left(\hat{\alpha}_{E}, \hat{\alpha}_{L}, \underline{\tau}_{O}\right)<0\end{cases}$

${ }^{10}$ Mueller-Frank and Pa: 2015), Proposition 1, derives a similar result under consumer homogeneity. 
- $\frac{\partial m}{\partial \underline{\tau}_{N}}= \begin{cases}\frac{1}{2}\left[\left(\hat{\alpha}_{E}-\frac{1}{2}\right)+\lambda\left(\hat{\alpha}_{L}-\frac{1}{2}\right)\right]>0 & \text { if } h\left(\hat{\alpha}_{E}, \hat{\alpha}_{L}, \underline{\tau}_{O}\right) \geq 0 \\ \frac{1}{2}\left(1+\lambda \underline{\tau}_{O}\right)\left(\hat{\alpha}_{E}-\frac{1}{2}\right)>0 & \text { if } h\left(\hat{\alpha}_{E}, \hat{\alpha}_{L}, \underline{\tau}_{O}\right)<0\end{cases}$

- $\frac{\partial m}{\partial \underline{\tau}_{O}}= \begin{cases}0 & \text { if } h\left(\hat{\alpha}_{E}, \hat{\alpha}_{L}, \underline{\tau}_{O}\right) \geq 0 \\ \frac{1}{2} \underline{\tau}_{N} \lambda\left(\hat{\alpha}_{E}-\frac{1}{2}\right)>0 & \text { if } h\left(\hat{\alpha}_{E}, \hat{\alpha}_{L}, \underline{\tau}_{O}\right)<0\end{cases}$

As to the sign of $\frac{\partial m}{\partial \underline{q}}$, we proceed as follows. Recall that, by definition, $\underline{\tau}_{N}=\tau_{N}(\underline{q})=$ $1-F_{c}\left(c_{N}(\underline{q})\right)=\int_{c_{N}(\underline{q})}^{1} f_{c}(x) d x$. Hence,

$$
\frac{\partial \underline{\tau}_{N}}{\partial c_{N}(\underline{q})}=\frac{\partial\left(\int_{c_{N}(\underline{q})}^{1} f_{c}(x) d x\right)}{\partial c_{N}(\underline{q})}=-f_{c}\left(c_{N}(\underline{q})\right)<0 .
$$

Applying the Leibniz integral rule,

$$
\frac{\partial c_{N}(\underline{q})}{\partial \underline{q}}=\frac{\partial\left(\int_{\underline{q}}^{1}(q-\underline{q}) f_{q}(q) d q\right)}{\partial \underline{q}}=-\int_{\underline{q}}^{1} f_{q}(q) d q<0 .
$$

Combining (12) and (13), it follows that

$$
\frac{\partial \underline{\tau}_{N}}{\partial \underline{q}}=\underbrace{\frac{\partial \underline{\tau}_{N}}{\partial c_{N}(\underline{q})}}_{<0} \underbrace{\frac{\partial c_{N}(\underline{q})}{\partial \underline{q}}}_{<0}>0
$$

Repeating the argument for $\underline{\tau}_{O}$,

$$
\frac{\partial \underline{\tau}_{O}}{\partial c_{O}(\underline{q})}=\frac{\partial\left(\int_{c_{O}(\underline{q})}^{1} f_{c}(x) d x\right)}{\partial c_{O}(\underline{q})}=-f_{c}\left(c_{O}(\underline{q})\right)<0
$$

and, since by definition $c_{O}(\underline{q})=\underline{\tau}_{N} \cdot c_{N}(\underline{q}), \frac{\partial c_{O}(\underline{q})}{\partial \underline{q}}=\frac{\partial c_{N}(\underline{q})}{\partial \underline{q}}\left[\frac{\partial \underline{\tau}_{N}}{\partial c_{N}(\underline{q})} \cdot c_{N}(\underline{q})+\underline{\tau}_{N}\right]$, which means that

$$
\frac{\partial c_{O}(\underline{q})}{\partial \underline{q}}=\underbrace{\frac{\partial c_{N}(\underline{q})}{\partial \underline{q}}}_{<0 \text { by } \underline{\underline{13}}} \underbrace{\left[\int_{c_{N}(\underline{q})}^{1} f_{c}(x) d x-c_{N}(\underline{q}) \cdot f_{c}\left(c_{N}(\underline{q})\right)\right]}_{>0}<0 .
$$

Combining (15) and (16),

$$
\frac{\partial \underline{\tau}_{O}}{\partial \underline{q}}=\underbrace{\frac{\partial \underline{\tau}_{O}}{\partial c_{O}(\underline{q})}}_{<0} \underbrace{\frac{\partial c_{O}(\underline{q})}{\partial \underline{q}}}_{<0}>0 .
$$


Moreover, by (14) and (17),

$$
\frac{\partial \underline{\tau}_{N} \underline{\tau}_{O}}{\partial \underline{q}}=\underbrace{\frac{\partial \underline{\tau}_{N}}{\partial \underline{q}}}_{>0} \cdot \underline{\tau}_{O}+\underbrace{\frac{\partial \underline{\tau}_{O}}{\partial \underline{q}}}_{>0} \cdot \underline{\tau}_{N}>0 .
$$

Finally, if $h\left(\hat{\alpha}_{E}, \hat{\alpha}_{L}, \underline{\tau}_{O}\right) \geq 0$, then by 14 , $\frac{\partial m}{\partial q}=\frac{1}{2}\left[\left(\hat{\alpha}_{E}-\frac{1}{2}\right)+\lambda\left(\hat{\alpha}_{L}-\frac{1}{2}\right)\right] \cdot \frac{\partial \underline{\tau}_{N}}{\partial q}>$ 0 , whereas if $h\left(\hat{\alpha}_{E}, \hat{\alpha}_{L}, \underline{\tau}_{O}\right)<0$, then 14 and 18 imply that $\frac{\partial m}{\partial \underline{q}}=\frac{1}{2}\left(\hat{\alpha}_{E}-\frac{1}{2}\right)$. $\left[\frac{\partial \underline{\tau}_{N}}{\partial \underline{q}}+\lambda \frac{\partial \underline{\tau}_{N} \underline{\tau}_{O}}{\partial \underline{q}}\right]>0$, which establishes the desired result.

\section{A.3 Proof of Proposition 3}

The probability that an arbitrary consumer $i \in N$ buys the product of the lowest quality firm is $P_{i N}^{B}\left(m^{A}, m^{B}\right)=\left[1-\rho\left(m^{A}, m^{B}\right)\right] \alpha_{i} \underline{\tau}_{N}+\rho\left(m^{A}, m^{B}\right)\left(1-\alpha_{i}\right) \underline{\tau}_{N}$. Likewise, an arbitrary consumer $j \in O$ that observes the purchase of an early consumer $k \in E$ buys good $B$ with probability $P_{j O}^{B}\left(m^{A}, m^{B}\right)=P_{k N}^{B}\left(m^{A}, m^{B}\right) \underline{\tau}_{O}$. Since by Proposition 1 the expenditures of the firms on display ad are equal in equilibrium, it follows that

$$
P_{i N}^{B}\left(m^{A}, m^{B}\right)=\frac{1}{2} \alpha_{i} \underline{\tau}_{N}+\frac{1}{2}\left(1-\alpha_{i}\right) \underline{\tau}_{N}=\frac{1}{2} \underline{\tau}_{N},
$$

and

$$
P_{j O}^{B}\left(m^{A}, m^{B}\right)=P_{k E}^{B}\left(m^{A}, m^{B}\right) \underline{\tau}_{O}=\frac{1}{2} \underline{\tau}_{N} \underline{\tau}_{O} .
$$

Moreover, $\underline{\tau}_{N}$ is almost surely strictly positive, and $\underline{\tau}_{O}$ is almost surely strictly smaller than one. ${ }^{11}$ Hence, given that $\underline{\tau}_{O}>\underline{\tau}_{N}$, by $(19)$ and $(20)$, for all $i \in N$ and $j \in O$,

$$
P_{j O}^{B}\left(m^{A}, m^{B}\right)<P_{i N}^{B}\left(m^{A}, m^{B}\right)<\frac{1}{2},
$$

and consequently

$$
\frac{1}{2}<\underbrace{1-P_{i N}^{B}\left(m^{A}, m^{B}\right)}_{=P_{i N}^{A}\left(m^{A}, m^{B}\right)}<\underbrace{1-P_{j O}^{B}\left(m^{A}, m^{B}\right)}_{=P_{j O}^{A}\left(m^{A}, m^{B}\right)} .
$$

In words, the expressions in (21) and (22) establish that (1) each consumer almost surely purchases the superior product with a strictly higher probability, and (2) receiving social information almost surely further increases (and respectively, decreases) the probability of buying the superior (and respectively, inferior) product.

\footnotetext{
${ }^{11}$ Indeed, $\underline{\tau}_{N}$ is equal to zero if and only if $\underline{q}=0$, which happens with probability zero. Likewise, $\underline{\tau}_{O}$ is equal to one if and only if either $q=0$ or $q=\overline{1}$, which happens again with probability zero.
} 


\section{A.4 Proof of Proposition 4}

From the definition of the (expected) social welfare in equation (5), it is immediate that

$$
\frac{\partial W(v)}{\partial v}=\lambda \cdot\left[\mathbb{E}\left(q_{a_{\ell}}-C_{L} \mid \ell \in O\right)-\mathbb{E}\left(q_{a_{\ell}}-C_{L} \mid \ell \in N \cap L\right)\right]
$$

Therefore, since $\lambda>0$, the sign of $\frac{\partial W(v)}{\partial v}$ depends on the difference between the late consumers' expected utilities, namely, $\mathbb{E}\left(q_{a_{\ell}}-C_{L} \mid \ell \in O\right)-\mathbb{E}\left(q_{a_{\ell}}-C_{L} \mid \ell \in N \cap L\right)$.

On the one hand, for those in the subgroup $O \subseteq L$ that observe social information,

$$
\begin{aligned}
& \mathbb{E}\left(q_{a_{\ell}}-\right.\left.C_{L} \mid \ell \in O\right)=P_{E}^{B} \cdot \tau_{O}\left(q^{B}\right) \cdot q^{B}+ \\
&+ P_{E}^{B} \cdot\left(1-\tau_{O}\left(q^{B}\right)\right) \cdot\left[q^{A}-\mathbb{E}\left(C_{L} \mid C_{L} \leq c_{O}\left(q^{B}\right)\right)\right]+ \\
& \quad+\left(1-P_{E}^{B}\right) \cdot\left[q^{A}-\mathbb{E}\left(C_{L} \mid C_{L} \leq c_{O}\left(q^{A}\right)\right)\right]+ \\
&+\left(1-P_{E}^{B}\right) \cdot \tau_{O}\left(q^{A}\right) \cdot \mathbb{E}\left(C_{L} \mid C_{L} \leq c_{O}\left(q^{A}\right)\right)
\end{aligned}
$$

On the other hand, for the subgroup of late consumers $N \cap L$ uninformed of the early buyers' purchases, the expected utility is

$$
\begin{gathered}
\mathbb{E}\left(q_{a_{\ell}}-C_{L} \mid \ell \in N \cap L\right)=P_{E}^{B} \cdot q^{B}+\left(\frac{1}{2}-P_{E}^{B}\right) \cdot\left[q^{A}-\mathbb{E}\left(C_{L} \mid C_{L} \leq c_{N}\left(q^{B}\right)\right)\right]+ \\
+\frac{1}{2} \cdot\left[q^{A}-\mathbb{E}\left(C_{L} \mid C_{L} \leq c_{N}\left(q^{A}\right)\right)\right]+\frac{1}{2} \cdot \tau_{N}\left(q^{A}\right) \cdot \mathbb{E}\left(C_{L} \mid C_{L} \leq c_{N}\left(q^{A}\right)\right) .
\end{gathered}
$$

Thus, combining (24) and (25) and after some algebraic manipulation, the difference of the expected utilities can be written as

$$
\begin{gathered}
\mathbb{E}\left(q_{a_{\ell}}-C_{L} \mid \ell \in O\right)-\mathbb{E}\left(q_{a_{\ell}}-C_{L} \mid \ell \in N \cap L\right)= \\
=P_{E}^{B} \cdot\left(1-\tau_{O}\left(q^{B}\right)\right) \cdot\left[q^{A}-q^{B}-\mathbb{E}\left(C_{L} \mid C_{L} \leq c_{O}\left(q^{B}\right)\right)\right]+ \\
+\left(\frac{1}{2}-P_{E}^{B}\right) \cdot \mathbb{E}\left(C_{L} \mid C_{L} \leq c_{N}\left(q^{B}\right)\right)+\frac{1}{2} \cdot\left(1-\tau_{N}\left(q^{A}\right)\right) \cdot \mathbb{E}\left(C_{L} \mid C_{L} \leq c_{N}\left(q^{A}\right)\right)- \\
-\left(1-P_{E}^{B}\right) \cdot\left(1-\tau_{O}\left(q^{A}\right)\right) \cdot \mathbb{E}\left(C_{L} \mid C_{L} \leq c_{O}\left(q^{A}\right)\right) .
\end{gathered}
$$

The relation established in Section 3 for the cutoff costs, namely, $c_{O}(q)<c_{N}(q)$ for all $q \in[0,1]$, implies that $\mathbb{E}\left(C_{L} \mid C_{L} \leq c_{O}(q)\right)<\mathbb{E}\left(C_{L} \mid C_{L} \leq c_{N}(q)\right)$; and by the same token, that $1-\tau_{O}(q)<1-\tau_{N}(q)$. In addition, recall that the cutoff cost $c_{N}(q)$ is decreasing in $q$ (i.e., the higher the quality of the product sampled first, the lower the probability for the buyers in $N$ to search further). Hence, for $q^{A}>q^{B}$, it follows that $\mathbb{E}\left(C_{L} \mid C_{L} \leq c_{N}\left(q^{A}\right)\right)<$ $\mathbb{E}\left(C_{L} \mid C_{L} \leq c_{N}\left(q^{B}\right)\right)$.

By definition, a late consumer $\ell \in O$ searches twice after observing a purchase of product 
$B$ if and only if $C_{L} \leq c_{O}\left(q^{B}\right)$. Since

$$
c_{O}\left(q^{B}\right)<c_{N}\left(q^{B}\right)=\int_{q^{B}}^{1}\left(q-q^{B}\right) d F_{q}(q)=\mathbb{E}\left(q-q^{B} \mid q>q^{B}\right)=\mathbb{E}\left(q^{A}-q^{B}\right)
$$

we have that $\mathbb{E}\left(C_{L} \mid C_{L} \leq c_{O}\left(q^{B}\right)\right)<\mathbb{E}\left(q^{A}-q^{B}\right)$, i.e., the expected cost incurred by the late consumer is outweighed by the expected quality gain.

Going back to the terms of equation (26), the expected utility difference between a socially-informed late consumer and an uninformed one is

$$
\begin{gathered}
\mathbb{E}\left(q_{a_{\ell}}-C_{L} \mid \ell \in O\right)-\mathbb{E}\left(q_{a_{\ell}}-C_{L} \mid \ell \in N\right)= \\
=P_{E}^{B} \cdot\left(1-\tau_{O}\left(q^{B}\right)\right) \cdot \underbrace{\left[\mathbb{E}\left(q^{A}-q^{B}\right)-\mathbb{E}\left(C_{L} \mid C_{L} \leq c_{O}\left(q^{B}\right)\right)\right]}_{>0}+ \\
+\left(\frac{1}{2}-P_{E}^{B}\right) \cdot \underbrace{\mathbb{E}\left(C_{L} \mid C_{L} \leq c_{N}\left(q^{B}\right)\right)}_{>\mathbb{E}\left(C_{L} \mid C_{L} \leq c_{O}\left(q^{A}\right)\right)}+\frac{1}{2} \cdot \underbrace{\left(1-\tau_{N}\left(q^{A}\right)\right)}_{>1-\tau_{O}\left(q^{A}\right)} \cdot \underbrace{\mathbb{E}\left(C_{L} \mid C_{L} \leq c_{N}\left(q^{A}\right)\right)}_{>\mathbb{E}\left(C_{L} \mid C_{L} \leq c_{O}\left(q^{A}\right)\right)}- \\
-\left(1-P_{E}^{B}\right) \cdot\left(1-\tau_{O}\left(q^{A}\right)\right) \cdot \mathbb{E}\left(C_{L} \mid C_{L} \leq c_{O}\left(q^{A}\right)\right) .
\end{gathered}
$$

Finally, from equation (27) it follows that almost surely

$$
\begin{aligned}
& \mathbb{E}\left(q_{a_{\ell}}-C_{L} \mid \ell \in O\right)-\mathbb{E}\left(q_{a_{\ell}}-C_{L} \mid \ell \in N\right)>\left(\frac{1}{2}-P_{E}^{B}\right) \cdot \mathbb{E}\left(C_{L} \mid C_{L} \leq c_{O}\left(q^{A}\right)\right)+ \\
& \quad+\frac{1}{2} \cdot\left(1-\tau_{O}\left(q^{A}\right)\right) \cdot \mathbb{E}\left(C_{L} \mid C_{L} \leq c_{O}\left(q^{A}\right)\right)-\left(1-P_{E}^{B}\right) \cdot\left(1-\tau_{O}\left(q^{A}\right)\right) \cdot \mathbb{E}\left(C_{L} \mid C_{L} \leq c_{O}\left(q^{A}\right)\right),
\end{aligned}
$$

which can be rewritten after some simplifications as

$$
\begin{aligned}
\mathbb{E}\left(q_{a_{\ell}}-C_{L} \mid \ell\right. & \in O)-\mathbb{E}\left(q_{a_{\ell}}-C_{L} \mid \ell \in N\right)> \\
& >\mathbb{E}\left(C_{L} \mid C_{L} \leq c_{O}\left(q^{A}\right)\right) \cdot\left[\left(\frac{1}{2}-P_{E}^{B}\right) \cdot \tau_{O}\left(q^{A}\right)\right]>0,
\end{aligned}
$$

where the last inequality follows from the fact that by $(19), P_{E}^{B}<1 / 2$. Therefore, from equations 23 and 28 , we conclude that almost surely $\frac{\partial W(v)}{\partial v}>0$, as desired. 